\title{
PREDICTING INDIVIDUAL DIFFERENCES IN CONFLICT DETECTION AND BIAS SUSCEPTIBILITY DURING REASONING
}

\author{
Jakub Šrol $^{\mathrm{a}}$ \& Wim De Neys ${ }^{\mathrm{b}}$

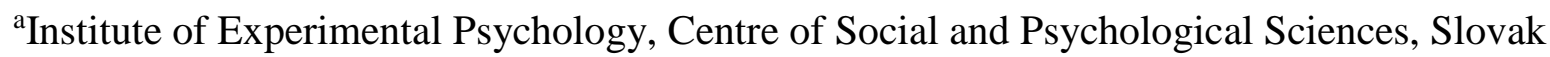 \\ Academy of Sciences \\ ${ }^{\text {b} P a r i s ~ D e s c a r t e s ~ U n i v e r s i t y, ~ L a P s y D E ~(U M R ~ C N R S ~ 8240), ~ P a r i s, ~ F r a n c e ~}$
}

MANUSCRIPT IN PRESS (THINKING \& REASONING)

Corresponding author:

Jakub Šrol

Institute of Experimental Psychology, Centre of Social and Psychological Sciences, Slovak Academy of Sciences

Dúbravská cesta 9, 84104 Bratislava, Slovakia

Phone: + 421 - 2 - 54775625

Email: jakub.srol@savba.sk

\section{ACKNOWLEDGEMENTS}

The study is based on an unpublished doctoral dissertation of the first author (J.S.). We would like to thank Maggie Toplak, Keith Stanovich, Valerie Thompson, and two anonymous reviewers for their helpful comments on an earlier draft of this manuscript. This study was supported by the Slovak Research and Development Agency and is part of the research project APVV-16-0153: “Cognitive failures - individual predictors and intervention possibilities". We would also like to thank the ANR for their support (ANR-16-CE28-001001). Data for this study are publicly available at OSF: https://osf.io/3cp9u/ 


\begin{abstract}
One of the key components of the susceptibility to cognitive biases is the ability to monitor for conflict that may arise between intuitively cued "heuristic" answers and logical principles. While there is evidence that people differ in their ability to detect such conflicts, it is not clear which individual factors are driving these differences. In the present large-scale study $(N=$ 399) we explored the role of cognitive ability, thinking dispositions, numeracy, cognitive reflection, and mindware instantiation (i.e. knowledge of logical principles) as potential predictors of individual differences in conflict detection ability and overall accuracy on a battery of reasoning problems. Results showed that mindware instantiation was the single best predictor of both conflict detection efficiency and reasoning accuracy. Cognitive reflection, thinking dispositions, numeracy, and cognitive ability played a significant but smaller role. The full regression model accounted for $40 \%$ of the variance in overall reasoning accuracy, but only $7 \%$ of the variance in conflict detection efficiency. We discuss the implications of these findings for popular process models of bias susceptibility.
\end{abstract}

Keywords: conflict detection, bias susceptibility, mindware instantiation, individual differences 


\section{PREDICTING INDIVIDUAL DIFFERENCES IN CONFLICT DETECTION AND BIAS SUSCEPTIBILITY DURING REASONING}

\section{INTRODUCTION}

Several decades of research in the reasoning and decision-making field have shown that even educated reasoners often violate basic logico-mathematical principles (Kahneman, 2011). In general, the problem seems to be that human reasoners have a strong tendency to base their inferences on intuitive rules-of-thumb or "heuristics". Although these intuitive "heuristic" responses will often cue valid problem solutions, they can also conflict with more logical considerations and bias our reasoning. To illustrate, consider the famous bat and ball problem: "A bat and a ball cost $\$ 1.10$. The bat costs $\$ 1.00$ more than the ball. How much does the ball cost?” (Frederick, 2005, p.27). Obviously, upon some reflection it is clear that the correct answer is "5 cents" (i.e., 5 cents ball $+\$ 1.05$ bat $=\$ 1.10$ ). However, most educated adults tend to answer that the ball costs 10 cents. The problem seems to be that people intuitively split the \$1.10 in \$1 and 10 cents and neglect the "more than" statement (De Neys, Rossi, \& Houdé, 2013). This intuitively cued "10 cents" answer seems to have an irresistible pull on people's thinking and leads them astray (Kahneman, 2011). And yet, some people are more successful than others at resisting the tendency to go with their heuristic answers when solving such problems, which is at least in part attributable to their ability to detect when their intuition conflicts with the logical consideration of the task at hand (e.g., Frey, Johnson, \& De Neys, 2018; Pennycook, Fugelsang, \& Koehler, 2015). In the present study, we set out to examine individual difference predictors of this conflict detection ability and their contribution to the overall accuracy on conflict reasoning problems.

Not surprisingly, many theoretical reasoning models have posited that the ability to detect the conflict between intuitively cued heuristic responses and logico-mathematical considerations is critical for sound reasoning (De Neys \& Bonnefon, 2013; Evans, 2007; Kahneman, 2011; Stanovich, 2018; Stanovich \& West, 2008). Notably, the conflict detection mechanism plays an integral role in traditional default-interventionist models of reasoning (Evans \& Stanovich, 2013; Kahneman, 2011; Stanovich \& West, 2008). Under such accounts, higher cognitive processes are thought to involve a sequential employment of two types of thought: intuitive (type 1) processing leads to a fast response based on heuristics and initial 
problem representation which reasoners can subsequently either affirm or try to correct by engaging in more cognitively demanding analytic (type 2) thought. When dealing with traditional reasoning tasks, such as the bat and ball problem above, the default-interventionist model assumes that intuitive thinking first produces a biased response and thus reasoners need to engage in analytic processing to suppress their intuition and make use of their explicit knowledge to derive the correct answer (Evans \& Stanovich, 2013). However, because of the computational costs of analytic thinking, when intuition leads to a response which is not in line with logical considerations of a task at hand, most people will not engage in type 2 processing and thus will not detect this intuition/logic conflict. Hence, according to the traditional default-interventionist model, people are often biased precisely because they fail to detect that their intuitions are in conflict with logical considerations of the task at hand (Kahneman, 2011).

Empirical research on the conflict detection arose precisely to test such predictions derived from the default-interventionist models. In a typical empirical study on conflict detection (for reviews see De Neys, 2012, 2013, 2017), participants are asked to solve reasoning problems from the heuristics and biases literature, as well as their no-conflict counterparts (see Table 1). The two types of tasks are constructed to be as similar as possible in regard to semantic content and their solution requires applying the same logical principles. The only intended difference between them is that while conflict problems are designed to cue heuristic intuitive responses which are in conflict with the solution based on logical norms, in no-conflict tasks intuitive thinking converges with the logical norm in question. The rationale behind conflict detection studies is that if biased reasoners are not detecting the conflict between the logical principle and the intuitively cued response, they would process the conflict problems in the same way as they do the no-conflict ones (De Neys \& Glumicic, 2008).

This is, however, not what the available evidence suggests. A large body of conflict detection studies shows that when people give heuristic responses on conflict versions of reasoning problems, they show decreased response confidence in comparison with the noconflict versions (Frey \& De Neys, 2017; Mevel et al., 2014; Stupple, Ball, \& Ellis, 2013), prolonged response times (Pennycook et al., 2015; Stupple \& Ball, 2008; Swan, Calvillo, \& Revlin, 2018), lower feelings of rightness about their answers (Thompson \& Johnson, 2014), better recall of information presented in the task (De Neys \& Glumicic, 2008), changes in skin conductance (De Neys, Moyens, \& Vansteenwegen, 2010), and other neurophysiological 
changes (Bago et al., 2018; De Neys, Vartanian, \& Goel, 2008; Vartanian et al., 2018). This seems to indicate that even when people are biased, they are at least implicitly sensitive to the fact that their response is not in line with the logically correct response (De Neys, 2012, 2017; however, for critics of this account see Mata, Ferreira, Voss, \& Kollei, 2017; Pennycook, Fugelsang, \& Koehler, 2012; Singmann, Klauer, \& Kellen, 2014).

Table 1. Conflict and no-conflict version of the conjunction fallacy task

\begin{tabular}{ll}
\hline \multicolumn{1}{c}{ Conflict version } & \multicolumn{1}{c}{ No-conflict version } \\
\hline $\begin{array}{l}\text { Bill is 34. He is intelligent, punctual but } \\
\text { unimaginative and somewhat lifeless. In school, } \\
\text { he was strong in mathematics but weak in social }\end{array}$ & $\begin{array}{l}\text { Bill is 34. He is intelligent, punctual but } \\
\text { unimaginative and somewhat lifeless. In school, } \\
\text { he was strong in mathematics but weak in social } \\
\text { studies and humanities. }\end{array}$ \\
$\begin{array}{l}\text { Which one of the following statements is most } \\
\text { likely? }\end{array}$ & $\begin{array}{l}\text { Which one of the following statements is most } \\
\text { likely? }\end{array}$ \\
$\begin{array}{l}\text { 1. Bill plays in a rock band for a hobby } \\
\text { 1. Bill is an accountant } \\
\text { 2. Bill is an accountant and plays in a rock band }\end{array}$ & $\begin{array}{l}\text { 2. Bill is an accountant and plays in a rock band } \\
\text { for a hobby }\end{array}$
\end{tabular}

(De Neys \& Bonnefon, 2013, p. 175)

Note. In the conflict version of the task, the stereotypical description cues the second option. However, choosing it is considered logically incorrect, as it violates the conjunction rule, i.e. likelihood of two events occurring simultaneously can never exceed the likelihood of one of them occurring separately (Tversky \& Kahneman, 1983). In the no-conflict problem, however, both the stereotypical description and the conjunction rule cue the first option.

Given the conflict detection findings, it appeared that people are quickly and effortlessly processing the logical structure of reasoning tasks to detect that their intuition is in conflict with it (De Neys, 2012, 2013, 2017). As these results were not a priori predicted by traditional default-interventionist models (e.g., Evans \& Stanovich, 2013; Kahneman, 2011; Stanovich \& West, 2008), several authors more recently moved on to advocate so-called hybrid dual process models (Bago \& De Neys, 2019a; De Neys \& Pennycook, 2019; Pennycook, et al., 2015; Thompson \& Newman, 2017). While the details of these accounts may differ, they all share the core idea that when people face conflict reasoning tasks, they quickly generate several intuitive responses based on heuristics as well as the logical structure of the problem. Differences in the relative strength of these intuitive outputs determine whether people will detect the conflict and subsequently engage in analytic type 2 processing.

Critically for the present research, early conflict detection studies have typically focused on group-level analyses which indicated that on average people are remarkably good 
at detecting conflict (De Neys \& Glumicic, 2008; Franssens \& De Neys, 2009). This has led researchers to believe that detection failures are unlikely to be a major source of individual differences in accurate reasoning (De Neys \& Bonnefon, 2013). Recently, however, the focus has shifted to a more individual-level approach and evidence emerged that people are not at all flawless in their detection ability. Indeed, across several studies, it was observed that at least $10-20 \%$ of participants did not show any signs of successful detection (Frey et al., 2018; Mevel et al., 2014; Pennycook et al., 2015). Moreover, this figure has been mostly obtained in studies with educated adults, therefore, one might expect that in the general population detection failures could be yet more prevalent. While there now seems ample evidence for substantial individual differences in conflict detection (Frey et al., 2018; Mata et al., 2017; Mevel et al., 2014; Pennycook et al., 2015; Swan et al., 2018), there is very little empirical research available that allows us to identify individual predictors related to the efficiency of this ability.

In theory, a crucial factor for successful conflict detection is the possession of specific mindware necessary to realize that one's intuition is not in line with logical considerations in the task at hand (De Neys \& Bonnefon, 2013; Stanovich, 2018). Mindware refers to stored knowledge of elementary mathematical and logical principles necessary for solving the traditional reasoning tasks (Stanovich \& West, 2008). Obviously, one will not be able to detect a conflict between an intuitively cued heuristic and a logical principle, if one doesn't know this principle. Recently, Stanovich (2018) has suggested that the degree of mindware instantiation (i.e. the degree to which activation of the principle is automatized) is a key factor in the success of conflict detection. Some preliminary evidence for this claim was already provided by Frey et al. (2018) who included in their research not only conflict and no-conflict tasks (such as those presented in Table 1), but also neutral versions of traditional reasoning problems which served as an indicator of participant's mindware instantiation. Neutral problems were designed to not cue any heuristic responses and thus accuracy on them depended primarily on participant's logico-mathematical knowledge (e.g., the impact of baserates on probability judgment). Yet, Frey et al. (2018) found mindware instantiation to be only moderately related to the conflict detection ability. This may, however, been in part due to the fact that they used a somewhat limited range of reasoning problems to measure both conflict detection and mindware instantiation (i.e. only base-rate neglect and conjunction fallacy tasks), thus restricting potential correlation between the two variables. 
It is also important to note that most of the previous research employed just one type of reasoning problem (e.g., base-rate neglect task), to study conflict detection. This presents a crucial drawback because, as was noted by Frey and De Neys (2017), it is not clear to what extent our detection ability is domain general. That is, we don't know whether people who successfully detect conflict on one problem are also more likely to detect it on other reasoning problems. Indeed, the authors showed that participant's detection ability was not significantly correlated across five different reasoning tasks. A related issue is whether people who show conflict detection as indicated by one index, e.g., decreased confidence, also exhibit detection on other indices. This was examined by Frey et al. (2018), who analyzed whether people consistently detect conflict across three measures: response latency, response confidence, and confidence latency. Their results draw attention to the fact that any single measure is an imperfect indicator of a person's detection ability, and that researchers should simultaneously utilize multiple indices in individual differences studies.

Taking these considerations into account, in the present study we made sure to examine individual differences in conflict detection while employing several indices and reasoning problems to measure participant's detection ability (Frey \& De Neys, 2017; Frey et al., 2018). As the available empirical evidence concerning individual variables specifically linked to the conflict detection is sparse, we also decided to examine - in addition to the degree of mindware instantiation - the contribution of a range of common individual difference predictors (i.e., cognitive ability, numeracy, cognitive reflection, and thinking dispositions, see method section for details) as these factors have been hypothesized to be potentially linked to conflict detection ability (Frey et al., 2018; Mevel et al., 2014; Stanovich, 2018). This will allow us to contrast the predictive potential of each individual factor.

In addition to predicting individual differences in conflict detection, the second goal of our study was to examine the role of detection ability and mindware instantiation in participants' overall accuracy on conflict reasoning problems. By employing several reasoning problems to measure participants' susceptibility to cognitive biases as well as a range of standard individual difference predictors, we were able to examine which factors contribute most to the reasoning performance. While individual difference research already established that cognitive ability, numeracy, cognitive reflection, and thinking dispositions all predict conflict reasoning accuracy (e.g., Klaczynski, 2014; Stanovich et al., 2016; Teovanović, Knežević, \& Stankov, 2015; Toplak, West, \& Stanovich, 2011), to our 
knowledge none of the studies have so far investigated these standard individual difference predictors along with indicators of both mindware instantiation and conflict detection ability. This could allow us to critically improve the reasoning accuracy predictions.

\section{METHOD}

\section{Participants}

Participants were recruited through the Prolific academic online service ${ }^{1}$ and paid $7.50 £$ for their participation. In total, 403 people took part in the study, however, four participants failed to correctly answer two or more of the attention check questions and were excluded from all subsequent analyses. Final sample consisted of 399 participants (32\% male, $66 \%$ female, $1 \%$ other) aged $18-73$ years $(M=35.81 ; S D=12.22)$. Most participants reported having some college degree ( $\sim 80 \%$ of the sample), $15 \%$ reported having a high school / GED education, and 2\% having less than high school education. The sample size of 400 participants was determined before the data collection began and amounted to the maximum number of participants we could recruit given our research budget. Sensitivity power analysis showed that a sample of this size should provide at least $80 \%$ power to detect any correlations of $r>.14$ with $5 \%$ error probability.

\section{Materials}

\section{Reasoning problems:}

Four types of reasoning problems were used in the study. For each type of task, there were four conflict, four no-conflict, and two neutral versions, resulting in 40 items in total. All reasoning problems are included in the supplementary materials. The neutral problems were used to compute the mindware instantiation index (see further).

Syllogistic reasoning task. In syllogisms, participants are presented with two premises and a conclusion and are asked to indicate whether the conclusion follows logically from the

\footnotetext{
${ }^{1}$ Participants were from United Kingdom, United States, Canada, Australia, or New Zealand, and all reported English as their first language. We do not have information about the distribution of different nationalities in our sample (participants were prescreened about their nationality but not asked about it further), however, most of the participant pool at Prolific academic online services are UK and US nationals (40\% and $30 \%$, respectively) at the time we are writing this (15.1.2019).
} 
premises under the assumption that the premises are true. In the conflict version of the task, the logical validity of a syllogism is in conflict with the believability of its conclusion (i.e. syllogism is either valid but unbelievable, or invalid but believable). No-conflict items were constructed by switching the minor premise and the conclusion and, in the case of unbelievable items, also changing the minor term of the syllogism (see De Neys et al., 2010). This was done to counterbalance problem content across conflict and no-conflict syllogisms. Items were based on the materials in De Neys et al. (2010). Two neutral items were also included which only dealt with abstract statements (i.e. "All $X$ are $Y$ "). Internal consistency for the four conflict items was $\alpha=.80$.

Base-rate neglect problems. Every problem provided two types of information about an imaginary person, a proportion of groups in the sample from which the person was randomly drawn (e.g., 5 engineers and 995 lawyers) and a stereotypical description of the individual, which cued one of the groups. Participants were asked to indicate to which of the two groups the imaginary person is more likely to belong. Two versions of the task were created by simply changing the base-rates to favor either the group in line with the stereotypical description (no-conflict items) or contrary to the stereotypical information (conflict problems). Neutral problems contained a description that did not favor any one of the groups from which the individual was randomly drawn. All items were based on materials used by De Neys and Glumicic (2008). The four conflict items showed good reliability $(\alpha=$ $.82)$.

Conjunction fallacy items. Participants were presented with a short stereotypical description of an individual following two statements from which they were supposed to choose the one which was the most likely. One statement always presented a single event pertaining to the described individual (e.g., "Jake plays the violin") and the other presented a conjunction of the first event with another feature (e.g., "Jake plays the violin and is jobless"). As the probability of the conjunctive statement can never exceed that of a single event, the single event option was always scored as correct. In no-conflict problems, this option contained the feature which was also representative of the described individual, while in conflict problems the representative feature was part of the conjunctive statement. Neutral problems simply assessed whether participants understood that the probability of a subset of events can never exceed the probability of a superset. Items were based on the material of Frey et al. (2018). Internal consistency of the four conflict items was $\alpha=.78$. 
Bat-and-ball problems. Conflict problems were based on the first problem of Frederick's (2005) Cognitive reflection test (“A bat and a ball ...”) but used different contents and numerical values. No-conflict versions were created by eliminating the "more than" statement from the original items (De Neys et al., 2013). In neutral problems, participants simply had to add the values for both items presented within the task. Items were based on the materials from Frey and De Neys (2017). Conflict items showed excellent reliability $(\alpha=.94)$.

\section{Conflict detection indices:}

Three measures were used as indicators of participants' conflict detection ability (Frey et al., 2018). First of all, the response latency from the onset of problem presentation until participants submitted a response was recorded. After submitting their response on a reasoning problem, participants were asked to rate their confidence in their answer on a scale of 1 ("not at all confident") to 11 ("absolutely confident"). The response confidence was used as a second index of conflict detection. Finally, the time that participants took to provide a confidence estimate was recorded and used as a third detection measure. Note that we will also combine the different detection indices in a composite (see results for details) and use it as a predictor of overall conflict reasoning accuracy.

In line with previous work, the conflict detection measures focus on the difference in latency and confidence between incorrectly solved conflict trials and correctly solved noconflict trials (De Neys et al., 2013; Frey et al., 2018; Mevel et al., 2014; Pennycook et al., 2015). The results for correct responses are not analyzed. Given that it is assumed that in case of correct responding reasoners also managed to block the heuristic response - and thereby resolved the conflict they initially detected - their response latency and confidence does not give us a pure indication of conflict detection efficiency per se (i.e. their initial doubt following conflict detection is also resolved, e.g., De Neys et al., 2013). This complicates the interpretation of conflict detection measures in case of correct responding. Finally, the rare trials in which no-conflict problems are solved incorrectly are discarded (e.g. De Neys \& Glumicic, 2008; Pennycook et al., 2015). Since in these problems both intuitive heuristic and logico-mathematical principle cue the correct response, it is hard to interpret incorrect responses on the no-conflict problem unequivocally.

\section{Mindware instantiation:}


Neutral versions of reasoning problems were used as a proxy measure of participant's mindware instantiation (Frey et al., 2018). Neutral tasks are similar to the conflict and noconflict problems but crucially they do not cue heuristic responses. In the absence of a heuristic response which would aid or hinder participant's reasoning, the accuracy on neutral problems depends mainly on the knowledge of logical principles necessary to solve the task at hand. In line with previous studies (e.g., Frey et al., 2018), the average accuracy on individual tasks was very high, as can be seen from Table 2 . For all analyses in the present study, we used overall accuracy on all neutral problems combined as an index of participant's mindware instantiation. The eight mindware instantiation items showed relatively poor reliability $(\alpha=$ .28), which was likely caused by very high performance on all neutral reasoning problems, and thus strong ceiling effect on these items. Furthermore, low reliability might also result from the fact that mindware instantiation is quite task specific (Stanovich, 2018).

Note that while mindware instantiation is used as a predictor throughout the analyses in the present study, it will be treated separately from the standard individual difference predictors due to its hypothesized distinct theoretical role in conflict detection and overall reasoning accuracy (De Neys \& Bonnefon, 2013; Stanovich, 2018).

Table 2. Accuracy on neutral reasoning problems

\begin{tabular}{lcc}
\hline & $M$ & $S D$ \\
\hline Syllogistic reasoning tasks & $71 \%$ & 28.93 \\
Base-rate neglect problems & $84 \%$ & 28.68 \\
Conjunction fallacy items & $73 \%$ & 38.43 \\
Bat-and-ball problems & $99.8 \%$ & 3.54 \\
Overall performance & $82 \%$ & 14.99 \\
\hline
\end{tabular}

Note. The table contains mean accuracies (in \%) and their standard deviations for the neutral versions of reasoning problems

Standard individual difference predictors:

Cognitive ability. To measure people's cognitive ability we used the Vienna matrix test (VMT; Klose, Černochová, \& Král, 2002). VMT is a standardized cognitive ability test which resembles the Raven's progressive matrices. The Czech adaptation of the VMT that was used in the present study shows a correlation of $r=.92$ with Raven' test (Klose et al., 2002). It originally consists of 24 items of increasing difficulty in which participants need to find a pattern in a complex $3 \times 3$ picture matrix and choose one of the eight options to complete it under a 24-minute time limit. To reduce participants load due to the study length, we decided to adopt a shortened, 14-item version with a 15-minute time limit based on the 
data collected in a previous study. Šrol (2019) showed that this shortened version has good reliability $(\alpha=.82)$ and a very high correlation with the full version of the test $(r=.96)$.

Thinking disposition measures. Thinking dispositions are related to people's epistemic values and self-regulation and entail propensities for different types of thought, such as the tendency to consider opposing views before reaching any conclusions, or to think extensively about a problem before responding (Stanovich, West, \& Toplak, 2016). To tap participants' analytic thinking disposition, a short 5-item Need for Cognition scale (NFC; example item: "I prefer complex to simple problems") was used. Similarly, we employed a 5-item Faith in Intuition scale (FI; example item: "I believe in trusting my hunches") to measure participants' inclination toward intuitive thinking. Both scales were taken from the work of Epstein, Pacini, Denes-Raj, \& Heier (1996). Epstein et al. report high correlations between both 5-item versions and their original longer counterparts ( $r=90$. for NFC; $r=.85$ for FI). In the present study, participants were asked to rate the items of both tests on a scale from 1 ("completely uncharacteristic of me") to 5 ("completely characteristic of me"). The NFC and FI were intended to reflect two independent processing styles rather than opposite ends of a single dimension (Epstein et al., 1996) and in line with this they tend to be uncorrelated (in the present study: $r(399)=-.09, p=.072)$. Therefore, in all analyses here we treated them as two separate individual difference predictors rather than a single thinking disposition composite.

Numeracy. Two methods were used to measure participants' numeracy. The first one was the Berlin numeracy test (BNT; Cokely, Galesic, Schulz, Ghazal, \& Garcia-Retamero, 2012), a four-item measure which in an extensive validation study showed good discrimination and convergent validity with other measures of numerical ability in diverse samples. As the open-ended version of the test is usually quite hard for use in the general population, a multiple-choice format of the BNT was used here (see Appendix in Cokely et al., 2012). Despite the multiple-choice format, the four-item test showed very low reliability in the present study $(\alpha=.41)$, presumably because most items showed up to be too hard for our participants $(M=1.62, S D=1.08)$. Second, we also included the self-report Subjective numeracy scale (SNS; Fagerlin et al., 2007). The SNS consists of 8 questions pertaining to the ability to use numerical information (e.g., "How good are you at working with fractions?") and preference for numerical over other formats of information (e.g., "How often do you find numerical information to be useful?") to which participants respond using a 6-point scale. In the present study, the scale exhibited very good reliability $(\alpha=.86)$. The average rating on the eight items was $3.88(S D=1.05)$. Because of the low reliability of BNT and moderate 
correlation between the two numeracy methods employed in the present study $(r=.25)^{2}$, we decided to normalize the scores on all 12 items of the two numeracy measures combined and compute the average of the normalized scores to create a single numeracy composite. This composite value is used as an index of the participant's numeracy throughout the study ${ }^{3}$.

Cognitive reflection measure $(C R)$. The cognitive reflection measure was modeled after Frederick's (2005) Cognitive Reflection Test. Four items were taken from Thomson and Oppenheimer (2016). Six additional items were based on Šrol (2019). The scores on the four and six item test were highly correlated $(r=.57)$ and were summed to form a single composite. Note that the bat-and-ball problem was not among the items.

The descriptive statistics for the standard individual difference measures are reported in Table 3.

Table 3. Descriptives of standard individual difference predictors

\begin{tabular}{lccc}
\hline & $M$ & $S D$ & $\alpha$ \\
\hline Cognitive ability - Vienna matrix test & 5.31 & 3.11 & .75 \\
Thinking dispositions - Need for Cognition & 3.50 & 0.77 & .79 \\
Thinking dispositions - Faith in Intuition & 3.65 & 0.76 & .87 \\
Numeracy & 0.00 & 0.56 & .81 \\
Cognitive reflection & 5.21 & 2.45 & .75 \\
\hline
\end{tabular}

Note. The table contains mean scores, standard deviations, and reliability estimates (Cronbach's $\alpha$ ) for the standard individual difference predictors employed in the study.

\section{Procedure}

The study was created with the Qualtrics software package and run online. It consisted of two blocks of materials, one containing the reasoning problems and the other containing the individual difference measures and one additional measure which is not reported in the present study. The order of the blocks was randomized between participants and the order of

${ }^{2}$ The low correlation between Berlin numeracy test scores and Subjective numeracy scale here is likely influenced by low reliability of the former method. For example, Fagerlin et al. (2007) report much stronger relationship between SNS and a different performance-based measure of numeracy $(r=.53)$.

3 To ensure that combining an objective and subjective numeracy measure into a single composite did not confound our results, we have also run all of the of analyses pertaining to individual difference predictors of conflict detection and conflict problem accuracy while including the two numeracy measures as separate variables. The analyses are available in Section $\mathrm{G}$ of the supplementary material. Note that the results are completely consistent with the key conclusions presented in the main manuscript. 
materials within each block was randomized within participants. Before every type of reasoning problem, participants were presented with instructions and an example item to familiarize them with the tasks. All reasoning problems except for bat-and-ball items were presented in two steps in order to reduce variability in response latencies due to reading (e.g., Frey et al., 2018). Participants first saw only the problem description, i.e. the first two premises of syllogisms, base-rate information in the base-rate neglect task, and the description of an individual in conjunction fallacy task. Then, the actual question was presented - the conclusion of a syllogism, description of an individual in base-rate neglect task, the two possible statements about the individual in the conjunction fallacy task - along with two response choices. Participants responded by selecting one of the response choices and submitted their response by clicking on a button to move to the next page. The time from the onset of the actual question presentation until they submitted their response was recorded. Participants were not explicitly told their responses to reasoning problems were timed but were instructed not to take breaks while solving these problems and to submit their answers immediately after deciding on the response. Three attention check questions were included in the study and participants who answered less than two of them correctly were automatically dropped from further analyses. Two attention check questions were mixed with base-rate neglect items and the cognitive reflection measure and were created to resemble these materials but to have an unambiguous correct response. One item was included in the BNT where participants were explicitly asked to always choose the option "none of the above" to indicate they had read the item.

\section{RESULTS}

The main aim of the present study was to identify individual predictors of reasoners' conflict detection ability and overall reasoning accuracy. However, for consistency with previous research, we first present the results of traditional conflict detection analyses where we examine the differences in participant's response latency, confidence, and confidence latency between conflict and no-conflict reasoning problems both in the entire sample (group-level analysis) and individually for participants who showed signs of successful conflict detection (individual-level analysis). We then explore the generality of the conflict detection ability by looking at the correlations between the ability to detect conflict across different detection indices and reasoning problems. Next, we conduct a correlation analysis to establish the 
mutual relationships between conflict detection efficiency, overall reasoning accuracy, mindware instantiation, and our standard individual difference predictors. Finally, we present two regression analyses in which we examine relative contributions of standard individual difference predictors and mindware instantiation to conflict detection efficiency and overall reasoning accuracy.

\section{Group-level reasoning accuracy and conflict detection analyses}

Table 4 shows an overview of the group-level reasoning accuracy and conflict detection findings. All results were analyzed separately for the four reasoning problems but for simplicity, we also calculated the overall performance across all conflict and no-conflict problems. Consistently with much previous research, overall accuracy for no-conflict problems $(M=93 \%, S D=8.62)$ was much higher than for their conflict $(M=43 \%, S D=$ 28.42) counterparts, $t(398)=35.54 ; p<.001 ; d=1.78$. As Table 4 shows, this pattern was observed on every individual reasoning task.

More importantly for the present study, for every participant we computed the difference in average response latency ${ }^{4}$, confidence, and confidence latency for incorrectly solved conflict and correctly answered no-conflict problems (e.g., Frey et al., 2018). Participants who did not give any incorrect answers on conflict or correct answers on the noconflict items were dropped from the respective analyses (n's are indicated in Table 4). Averaged across all reasoning problems, participants took longer to answer conflict problems than the no-conflict ones, $t(384)=11.64 ; p<.001 ; d=0.59$; and the former were associated with lower response confidence that the latter, $t(384)=14.17 ; p<.001 ; d=0.72$. By and large, the overall results were observed on each separate task. While there was no difference in overall confidence latency observed on conflict and no-conflict problems, $t(384)=0.06 ; p=$ $.95 ; d=0.00$; participants took significantly longer to provide confidence estimates for conflict syllogisms and bat-and-ball tasks. As is evident from Table 4, participants showed classic conflict detection signs in most of the individual reasoning problems and detection

\footnotetext{
${ }^{4}$ Prior to the analyses, all latency data were checked for outlying observations. Latency values which were more than three standard deviations above/below the mean of the respective index were replaced with the value of three standard deviations above/below the average. All analyses reported in the main manuscript and the supplementary material are based on the outlier treated data. However, all analyses were also run on the raw data (before outlier replacement) and the results were consistent with the conclusions presented in the study.
} 
indices. Exceptions were response confidence in the syllogistic reasoning task and confidence latency in base-rate and conjunction fallacy problems.

Table 4. Summary of group-level reasoning accuracy and conflict detection analyses

\begin{tabular}{|c|c|c|c|c|}
\hline & Accuracy & Response latency & $\begin{array}{l}\text { Response } \\
\text { confidence }\end{array}$ & $\begin{array}{c}\text { Confidence } \\
\text { latency }\end{array}$ \\
\hline \multicolumn{5}{|c|}{ Syllogistic reasoning task } \\
\hline no-conflict $(S D)$ & $86 \%(18.86)$ & $4.78(2.84)$ & $9.98(1.43)$ & $2.46(1.16)$ \\
\hline conflict $(S D)$ & $55 \%(38.74)$ & $5.52(4.20)$ & $9.67(1.65)$ & $2.66(1.40)$ \\
\hline \multirow[t]{2}{*}{ difference $(n=270)$} & $t(398)=16.60 * * *$ & $t(269)=3.30 * *$ & $t(269)=1.43$ & $t(269)=2.20 *$ \\
\hline & $d=0.83$ & $d=0.20$ & $d=0.09$ & $d=0.13$ \\
\hline \multicolumn{5}{|l|}{ Bat-and-ball items } \\
\hline no-conflict $(S D)$ & $98 \%(10.51)$ & $8.48(4.10)$ & $10.65(0.84)$ & $2.31(1.36)$ \\
\hline conflict $(S D)$ & $42 \%(45.68)$ & $12.21(8.92)$ & $10.05(1.92)$ & $2.61(1.68)$ \\
\hline \multirow[t]{2}{*}{ difference $(n=273)$} & $t(398)=22.67 * * *$ & $t(272)=8.30 * * *$ & $t(272)=5.71 * * *$ & $t(272)=2.61 *$ \\
\hline & $d=1.13$ & $d=0.50$ & $d=0.35$ & $d=0.16$ \\
\hline \multicolumn{5}{|c|}{ Base-rate neglect problems } \\
\hline no-conflict $(S D)$ & $94 \%(13.16)$ & $10.87(5.75)$ & $8.96(1.58)$ & $2.67(1.20)$ \\
\hline conflict $(S D)$ & $47 \%(39.95)$ & $12.70(7.99)$ & $7.78(1.94)$ & $2.65(1.21)$ \\
\hline \multirow[t]{2}{*}{ difference $(n=294)$} & $t(398)=23.57 * * *$ & $t(293)=5.03 * * *$ & $t(293)=9.47 * * *$ & $t(293)=0.33$ \\
\hline & $d=1.18$ & $d=0.29$ & $d=0.55$ & $d=0.02$ \\
\hline \multicolumn{5}{|c|}{ Conjunction fallacy problems } \\
\hline no-conflict $(S D)$ & $93 \%(16.85)$ & $7.89(4.15)$ & $7.96(2.10)$ & $2.79(1.20)$ \\
\hline conflict $(S D)$ & $30 \%(35.06)$ & $10.80(6.11)$ & $6.79(2.17)$ & $2.70(1.08)$ \\
\hline \multirow[t]{2}{*}{ difference $(n=341)$} & $t(398)=35.50 * * *$ & $t(340)=10.56 * * *$ & $t(340)=13.92 * * *$ & $t(340)=1.79$ \\
\hline & $d=1.68$ & $d=0.57$ & $d=0.75$ & $d=0.10$ \\
\hline \multicolumn{5}{|l|}{ Overall performance } \\
\hline no-conflict $(S D)$ & $93 \%(8.62)$ & $7.92(3.25)$ & $9.39(1.06)$ & $2.66(3.12)$ \\
\hline conflict $(S D)$ & $43 \%(28.42)$ & $10.49(5.54)$ & $8.28(1.71)$ & $2.65(1.11)$ \\
\hline \multirow[t]{2}{*}{ difference $(n=385)$} & $t(398)=35.54 * * *$ & $t(384)=11.64 * * *$ & $t(384)=14.17 * * *$ & $t(384)=0.06$ \\
\hline & $d=1.78$ & $d=0.59$ & $d=0.72$ & $d=0.00$ \\
\hline
\end{tabular}

\section{Individual-level conflict detection analyses}

Following Frey et al. (2018), for every detection index, participants who got at least one conflict item incorrect and one no-conflict item correct (whole biased group) were further divided into three subgroups according to whether they showed longer latencies and lower confidence for incorrect conflict than correct no-conflict problems (detection subgroup), the opposite pattern of results (reverse detection), or the same latency and confidence estimates for the two versions of problems (same subgroup). Here we only present the overview of results for the detection subgroup but a complete summary of individual-level conflict detection analyses can be found in the supplementary material. Table 5 presents the proportions of participants who successfully detected conflict, as well as the detection effects (i.e., the average difference in response latency, confidence, and confidence latency between 
conflict and no-conflict problems) across the reasoning problems and detection indices. Results were analyzed separately for the four reasoning problems but we again also calculated overall performance across all conflict and no-conflict problems for simplicity.

Considering the overall performance on all conflict and no-conflict reasoning problems combined, across the three indices $54-81 \%$ of biased reasoners showed signs of successful conflict detection. This high prevalence of successful detection was also observed in most of the individual reasoning tasks. Two exceptions were found in case of the response confidence index in syllogistic reasoning and bat-and-ball problems, where the proportion of participants exhibiting successful detection was somewhat lower. In sum, consistent with other studies that employed individual-level conflict detection analyses (Frey et al., 2018; Mevel et al., 2014; Pennycook et al., 2015) our results show that while on average most reasoners may be quite capable of detecting the misleading nature of their intuitions, substantial individual differences can nevertheless be observed on particular reasoning problems and detection indices.

Table 5. Summary of individual-level conflict detection analysis for the detection subgroup

\begin{tabular}{lccc}
\hline & \multicolumn{3}{c}{ Detection index } \\
\cline { 2 - 4 } & $\begin{array}{c}\text { Response } \\
\text { latency }\end{array}$ & $\begin{array}{c}\text { Response } \\
\text { confidence }\end{array}$ & $\begin{array}{c}\text { Confidence } \\
\text { latency }\end{array}$ \\
\hline $\begin{array}{l}\text { Syllogistic reasoning task } \\
\text { proportion of biased group }(n=270)\end{array}$ & $153(57 \%)$ & $97(36 \%)$ & $146(54 \%)$ \\
$\quad$ conflict detection effect $(S D)$ & $-2.75(3.38)$ & $-1.50(1.33)$ & $-0.99(1.44)$ \\
Bat-and-ball items & & & \\
$\quad$ proportion of biased group $(n=273)$ & $208(76 \%)$ & $79(29 \%)$ & $152(56 \%)$ \\
$\quad$ conflict detection effect $(S D)$ & $-5.78(7.11)$ & $-2.16(2.43)$ & $-1.13(1.77)$ \\
$\quad$ Base-rate neglect problems & & & \\
$\quad$ proportion of biased group $(n=294)$ & $169(57 \%)$ & $188(64 \%)$ & $140(48 \%)$ \\
$\quad$ conflict detection effect $(S D)$ & $-4.97(6.25)$ & $-2.05(1.60)$ & $-0.72(0.85)$ \\
Conjunction fallacy problems & & & \\
$\quad$ proportion of biased group $(n=341)$ & $268(79 \%)$ & $257(75 \%)$ & $168(49 \%)$ \\
$\quad$ conflict detection effect $(S D)$ & $-4.44(4.37)$ & $-1.67(1.29)$ & $-0.59(0.60)$ \\
Overall performance & & & \\
$\quad$ proportion of biased group $(n=384)$ & & & $208(54 \%)$ \\
$\quad$ conflict detection effect $(S D)$ & $311(81 \%)$ & $-1.22(1.13)$ & $-0.64(0.98)$ \\
\hline
\end{tabular}

Note. Response latency data are reported in seconds.

\section{Correlations of detection efficiency across detection indices \& reasoning problems}

To find out whether people consistently detected conflict across the three detection indices, we have computed their detection efficiency separately for response latency, response 
confidence, and confidence latency measures. The detection efficiency was calculated for every participant as the number of times they showed a successful detection on a given index divided by the total number of reasoning tasks on which they were biased (Frey \& De Neys, $2017)^{5}$. We calculated the amount of successful detections by summing the number of times participants showed either lower confidence, longer response latency, or longer confidence latency on the conflict in comparison with no-conflict versions of the four reasoning problems. As successful detection is calculated only from reasoning problems on which participants are biased and respond incorrectly, those problems on which a participant did not give any incorrect conflict responses were not used to calculate their detection efficiency. Therefore, we divided the amount of successful detections for every given participant by the total number of times they could have detected the conflict on the four reasoning problems (i.e. the number of times they answered incorrectly). Participants who did not give any incorrect responses to conflict problems $(n=15)$ were dropped from subsequent analyses.

The detection efficiency index calculated on the basis of response latencies was correlated with the one based on confidence, $r(384)=.238, p<.001$, but not with the one based on confidence latencies, $r(384)=.067, p=.19$. Confidence and confidence latency efficiencies were weakly correlated, $r(384)=.116, p=.02$. Given that confidence latency detection efficiency was at best weakly related to the other indices and previous research already questioned the reliability of this index (Frey et al., 2018), we decided to drop confidence latency from all subsequent analyses and focus on the two remaining indices (confidence and response latency) in the rest of the results. For completeness, the analyses pertaining to the confidence latency index can be found in the supplementary material.

While the abovementioned results show some consistency in successful detection based on response latency and confidence detection indices, we were also interested in whether participants' detection ability is related across different reasoning problems. To explore this we again computed detection efficiency for every participant, but this time

\footnotetext{
${ }^{5}$ For our present analyses we have chosen a categorical detection index approach, i.e. participants' detection efficiency was calculated by summing up the number of times they showed the detection effect. However, some authors (e.g., Pennycook et al., 2015) favor a continuous approach to the conflict detection measurement based on the size of the detection effect. Therefore, to please all readers regardless of the approach they prefer, all of the subsequent analyses were also repeated with detection effect sizes instead of detection efficiency indices. The results are presented in the supplementary material (see Tables S4, S5, and S6). Note that despite the external dissimilarity of the two approaches, by and large, they point to very similar conclusions.
} 
separately for every type of reasoning problem on which the participant was biased. Detection efficiency was calculated as the number of detected conflicts in a given task based on the response latency and confidence index divided by the number of detection indices. Correlation analysis showed that these indices were mostly unrelated, with detection efficiency in the bat-and-ball task showing no relation to detection efficiency in the base-rate, $r(219)=.059, p=.38$, or conjunction fallacy task, $r(242)=-.001, p=.99$. The efficiencies on the latter two were also not correlated, $r(278)=.044, p=.47$. The only significant correlation was between detection efficiency in syllogisms and base-rate problems, $r(225)=.133, p=$ .047. But again, the former was unrelated both to the bat-and-ball task, $r(212)=.031, p=.65$, and conjunction items, $r(247)=-.053, p=.41$. Hence, consistent with the findings of Frey and De Neys (2017), we have found very little evidence for the domain generality of conflict detection. Thus, even if people are quite successful in detecting conflicts within one type of a reasoning problem, this ability does not necessarily seem to transfer to another type of reasoning problem. For an interested reader, we also computed correlations between successful detection observed on every reasoning problem and every detection index separately, which can be found in the supplementary materials.

Note that for completeness, we also calculated reliability estimates for the key detection efficiency indices based on the response latency and confidence index. Results indicated that these were very low (response latency index: $\alpha=.03$, confidence index: $\alpha=.14$, detection efficiency for both indices combined: $\alpha=.25$ ). This undoubtedly reflects the nature of conflict detection measurement and low domain generality of detection ability ${ }^{6}$.

\section{Predicting individual differences in detection efficiency and conflict reasoning accuracy: correlations}

We now move to the critical question of how the standard individual difference predictors and mindware instantiation (i.e. average accuracy on neutral versions of reasoning problems) are related to participant's conflict detection efficiency and overall reasoning

\footnotetext{
${ }^{6}$ But reliability estimates are presumably also low because participants differed in the number of indices (i.e. number of tasks on which they were biased). Only those participants who were biased on all tasks could be included in the analysis $(n=176)$. We note that caution is required in interpreting indices with very low reliability, which is also likely a cause of the lower predictive power of these indices in our regression analyses. We come back to this issue in the discussion.
} 
accuracy. Although the detection efficiencies based on response latency and confidence were correlated, the relationship was not very strong. To avoid spurious conclusions we therefore decided to run the individual difference predictor analyses separately for the two detection measures, rather than to combine them into a single index.

First, we have examined the correlations between the two detection efficiencies, standard individual difference predictors, and mindware instantiation. Table 6 gives an overview of the correlation analyses. As the table shows, participants with a higher latency detection efficiency score higher on Need for Cognition, numeracy, and cognitive ability, although the relationships with this detection index were all relatively weak. Stronger correlations were found in the case of confidence detection efficiency, which was also related to participants' cognitive reflection and mindware instantiation. While both of the latter factors as well as an NFC-like thinking disposition measure were already shown to correlate with the conflict detection ability in certain reasoning problems (Frey et al., 2018; Pennycook et al., 2014, 2015), neither cognitive ability nor numeracy were previously observed to contribute to the detection efficiency. This may have been due to the fact that their relationship with conflict detection is relatively weak and remained undetected in the less highly powered previous studies.

Next, we examined whether standard individual difference predictors, mindware instantiation, and detection efficiency are related to overall conflict reasoning accuracy. For the purpose of this as well as all of the subsequent analyses, we have computed a single conflict reasoning accuracy composite score by summing the correct answers on all sixteen conflict reasoning problems $(\alpha=.87)^{7,8}$. Results are included in Table 6 . In line with previous research (Klaczynski, 2014; Teovanović et al., 2015; Toplak et al., 2011), the conflict reasoning accuracy composite showed moderate to strong correlations with all of the standard

\footnotetext{
${ }^{7}$ However, the results for every type of reasoning problem separately can be found in the supplementary material

${ }^{8}$ Previous research has shown that correlations between various reasoning problems tend to be relatively modest (e.g. Teovanović et al., 2015) and composite scores derived from larger set of different reasoning problems tend to show low internal consistency (Toplak et al., 2011). In contrast, correlations among reasoning problem accuracies in the present study (Table S2 in the supplementary material) range between .20-.46 and reliability for conflict reasoning accuracy composite is high. We believe this is because we did not employ large set of different reasoning problems, but rather four different types of problems with more items per problem type. This allowed creating reliable composites for every problem type which have shown enough commonality to be summed into a single composite (for a similar approach, see Klaczynski, 2014).
} 
individual difference predictors. There was no relationship between latency detection efficiency and conflict reasoning accuracy, but both confidence detection efficiency and mindware instantiation did correlate substantially with reasoning performance.

Table 6. Correlations between latency and confidence detection efficiency, conflict reasoning accuracy composite, standard individual difference predictors, and mindware instantiation

\begin{tabular}{lllllllll}
\hline & 1. & 2. & 3. & 4. & 5. & 6. & 7. & 8. \\
\hline 1. Detection efficiency: latency & 1 & & & & & & & \\
2. Detection efficiency: confidence & $\mathbf{. 2 4}$ & 1 & & & & & \\
3. Conflict reasoning accuracy & .07 & $\mathbf{. 2 7}$ & 1 & & & & \\
4. Cognitive reflection & .07 & $\mathbf{. 2 2}$ &. $\mathbf{4 7}$ & 1 & & & & \\
5. Faith in Intuition & .00 & -.03 & $\mathbf{- . 2 6}$ & $\mathbf{- . 1 2}$ & 1 & & \\
6. Need for Cognition & $\mathbf{. 1 4}$ & $\mathbf{. 1 0}$ & $\mathbf{. 2 4}$ & $\mathbf{. 2 2}$ & -.09 & 1 & & \\
7. Numeracy & $\mathbf{. 1 2}$ & $\mathbf{. 1 9}$ & $\mathbf{. 4 8}$ & $\mathbf{. 4 0}$ & $\mathbf{- . 1 1}$ & $\mathbf{. 3 8}$ & 1 & \\
8. Cognitive ability & $\mathbf{. 1 2}$ & $\mathbf{. 1 9}$ & $\mathbf{. 4 6}$ & $\mathbf{. 5 0}$ & $\mathbf{- . 1 9}$ & $\mathbf{. 2 5}$ & .48 & 1 \\
9. Mindware & .04 & $\mathbf{. 2 2}$ & $\mathbf{. 4 9}$ & $\mathbf{. 3 5}$ & $\mathbf{- . 1 5}$ & $\mathbf{. 2 1}$ & $\mathbf{. 3 4}$ & $\mathbf{. 3 5}$ \\
\hline
\end{tabular}

Note. Correlations pertaining to detection efficiencies are based on 384 observations, others on 399 observations. Correlations that appear in bold are significant at $p<.05$.

While the examination of correlations between mindware instantiation and standard individual difference predictors was not among the main aims of this study, we noticed some interesting trends in this regard which we briefly mention here. The role of mindware instantiation in both conflict detection and reasoning accuracy is theoretically acknowledged (Pennycook et al., 2015; Stanovich, 2018), yet, in empirical studies it rarely shows up to substantially contribute to reasoning performance (e.g., Frey \& De Neys, 2017; Frey et al., 2018). This is presumably because the participant's performance on neutral versions of reasoning problems, which is used as a proxy for their mindware, is usually very high. In the present study, however, while the average performance on neutral problems was also high, it was strongly correlated with the conflict reasoning accuracy composite and was also related to detection efficiency based on confidence. Moreover, mindware instantiation also showed moderate correlations with all of the standard individual difference measures. Such results suggest that even though the variability in available mindware may not be very large among educated adults, individual differences in mindware instantiation may still play a nonnegligible role in detection efficiency and conflict reasoning accuracy and may be a more important contributor to the reasoning performance than previously reckoned. 


\section{Predicting individual differences in detection efficiency: regression models}

We now turn to our key analyses. While mindware instantiation and several standard individual difference predictors were related to the latency and confidence detection efficiency indexes, these variables were themselves all moderately intercorrelated. Thus, to determine which of these factors are the strongest independent predictors of conflict detection, we conducted two regression analyses separately for latency and confidence detection efficiency. The results are summarized in Table 7. In the first regression, NFC showed up to be the only significant predictor $(\beta=.11)$ of the latency detection efficiency index when all other standard individual difference predictors and mindware instantiation were taken into account. However, the proportion of explained variance was very small (1.4\%) and the overall model was only marginally significant.

Table 7. Summary of the regression analysis predicting latency and confidence detection efficiency

\begin{tabular}{|c|c|c|c|c|}
\hline & \multicolumn{2}{|c|}{ Detection efficiency: latency } & \multicolumn{2}{|c|}{ Detection efficiency: confidence } \\
\hline & $\beta$ & $p$ & $\beta$ & $p$ \\
\hline Constant & & .001 & & .316 \\
\hline Mindware & -.02 & .706 & .14 & .011 \\
\hline Cognitive ability & .08 & .226 & .05 & .404 \\
\hline Numeracy & .05 & .442 & .07 & .243 \\
\hline Need for Cognition & .11 & .048 & .01 & .858 \\
\hline Faith in Intuition & .03 & .558 & .02 & .661 \\
\hline \multirow[t]{2}{*}{ Cognitive reflection } & .00 & .964 & .12 & .040 \\
\hline & \multicolumn{2}{|c|}{$R^{2}=.01, F(6,377)=1.91, p=.078$} & \multicolumn{2}{|c|}{$R^{2}=.07, F(6,377)=5.61, p<.001$} \\
\hline
\end{tabular}

Note. The table contains standardized regression coefficients $(\beta)$ with their respective significance. $R^{2}$ denotes adjusted $r$-square for the model with appropriate $F$-statistics. Significant regression coefficients are presented in bold.

In the second regression, both cognitive reflection $(\beta=.12)$ and mindware instantiation $(\beta=.14)$ predicted confidence detection efficiency after accounting for other variables in the regression. While the predictors explained more variance in case of the confidence detection index (7\%), their contributions were relatively weak and leave a lot of space for other potential predictors of the conflict detection ability. Interestingly, even though Need for Cognition, numeracy, and cognitive ability were related to confidence detection efficiency in the correlation analysis above, they did not show up as significant independent predictors in the regression model. Thus, their contribution to conflict detection might be primarily caused by their relationship with cognitive reflection, which has been shown to tap all three of the abovementioned factors (e.g., Thomson \& Oppenheimer, 2016). 


\section{Predicting individual differences in conflict reasoning accuracy: regression model}

While the correlations presented earlier showed that detection efficiency, mindware instantiation, and standard individual difference predictors are all related to the overall accuracy in reasoning problems, we also examined these variables as independent predictors in a linear regression on the conflict reasoning accuracy composite. In the first step of the regression, we entered all standard individual difference predictors. Then, we included mindware instantiation, and at the final step we entered both detection efficiency indices to the regression model. This approach was chosen to examine whether mindware instantiation and detection efficiency, both theoretically important determinants of bias susceptibility (Pennycook et al., 2015; Stanovich, 2018), predict reasoning accuracy over and above cognitive ability, thinking dispositions, numeracy, and cognitive reflection measures. The results are summarized in Table 8 .

All variables in the final model except for Need for Cognition and latency detection efficiency did significantly contribute to conflict reasoning accuracy. Among the standard individual difference predictors, numeracy $(\beta=.22)$, cognitive reflection $(\beta=.19)$, Faith in Intuition $(\beta=-.14)$, and cognitive ability $(\beta=.11)$ were found to independently predict the accuracy on conflict problems at the last step of the model. Overall, these standard individual difference predictors explained $34 \%$ of the variance in the conflict reasoning accuracy composite. Our results in this respect are consistent with previous individual difference predictor analyses (Klaczynski, 2014; Toplak et al., 2011). More critically, we examined whether mindware instantiation and conflict detection efficiency play a role in reasoning accuracy over and above standard individual difference predictors. At the second step of the regression, mindware instantiation accounted for $5 \%$ of additional variance over the standard individual difference measures and ended up being the strongest independent predictor $(\beta=$ .23) of conflict reasoning accuracy composite in the final model. Lastly, while the predictive power of confidence detection efficiency was not overly strong $(\beta=.11)$, it did show up as an independent predictor at the final step of the regression and it accounted for another $1 \%$ of the variance in accuracy on conflict problems after all of the other variables were accounted for. Thus, despite the substantial proportion of variance in conflict reasoning accuracy which was already explained by standard individual difference predictors, both mindware instantiation and confidence detection efficiency further contributed to the reasoning performance, in line with their hypothesized distinct theoretical role as key determinants of bias susceptibility. 
Together the variables explained approximately $40 \%$ of the variance in the conflict reasoning accuracy composite.

Table 8. Summary of the regression analysis predicting the composite of correctly answered conflict reasoning problems

\begin{tabular}{|c|c|c|}
\hline & $\beta$ & $p$ \\
\hline \multicolumn{3}{|l|}{ Step 1} \\
\hline Constant & & $<.001$ \\
\hline Cognitive ability & .15 & .004 \\
\hline Numeracy & .27 & .001 \\
\hline Need for Cognition & .03 & .524 \\
\hline Faith in Intuition & -.15 & $<.001$ \\
\hline Cognitive reflection & .26 & $<.001$ \\
\hline \multicolumn{3}{|c|}{$R^{2}=.34, F(5,378)=40.98, p<.001$} \\
\hline \multicolumn{3}{|l|}{ Step 2} \\
\hline Constant & & .690 \\
\hline Cognitive ability & .11 & .026 \\
\hline Numeracy & .23 & $<.001$ \\
\hline Need for Cognition & .01 & .763 \\
\hline Faith in Intuition & -.13 & .001 \\
\hline Cognitive reflection & .21 & $<.001$ \\
\hline Mindware & .25 & $<.001$ \\
\hline \multicolumn{3}{|c|}{$\Delta R^{2}=.05, F(1,377)=31.67, p<.001$} \\
\hline \multicolumn{3}{|l|}{ Step 3} \\
\hline Constant & & .733 \\
\hline Cognitive ability & .11 & .031 \\
\hline Numeracy & .22 & $<.001$ \\
\hline Need for Cognition & .01 & .745 \\
\hline Faith in Intuition & -.14 & .001 \\
\hline Cognitive reflection & .19 & $<.001$ \\
\hline Mindware & .23 & $<.001$ \\
\hline Detection efficiency: LAT & -.02 & .640 \\
\hline $\begin{array}{r}\text { Detection efficiency: CON } \\
\Delta R^{2}=.01, F(2,375)=3.5\end{array}$ & .11 & .008 \\
\hline
\end{tabular}

Note. The table contains standardized regression coefficients $(\beta)$ with their respective significance. $R^{2}$ and $\Delta R^{2}$ denote adjusted r-square for the initial model and change in r-square at the $2^{\text {nd }}$ and $3^{\text {rd }}$ step of the regression with appropriate change statistics. LAT: latency, CON: confidence. Significant regression coefficients are presented in bold. 


\section{Discussion}

In the present study, we set out to examine individual differences in the ability to detect intuition/logic conflict and people's overall reasoning accuracy while employing several traditional reasoning problems and detection indices to ensure the robustness of our results. We found that the Need for Cognition thinking disposition, mindware instantiation, and cognitive reflection are independent predictors of participants' detection ability, although the overall explained variance was quite low. Our results also show that detection efficiency and mindware instantiation are both predictors of the accuracy on conflict reasoning problems over and above the measures of cognitive ability, thinking dispositions, numeracy, and cognitive reflection. This is consistent with the hypothesized theoretical role of conflict detection and mindware instantiation as essential processes in the susceptibility to cognitive biases (De Neys \& Bonnefon, 2013; Pennycook et al., 2015; Stanovich, 2018).

Our key finding is that although various standard individual difference predictors played a significant role, the single best predictor of both conflict detection efficiency and overall reasoning accuracy turned out to be mindware instantiation. Theoretically, the availability of the necessary mindware that allows one to grasp the normative solution of the task at hand is thought to be one of the key factors for successful conflict detection as well as overall bias susceptibility (De Neys \& Bonnefon, 2013; Stanovich, 2018). Yet, mindware instantiation is often neglected in empirical studies on rational thinking because it is presumed that most educated adults do possess the basic rules of logic and mathematics necessary for solving the traditional reasoning tasks. This assumption seems to be supported by the almost perfect accuracy on neutral versions of such problems (e.g., De Neys \& Glumicic, 2008; Frey $\&$ De Neys, 2017; Frey et al., 2018). While we have also observed high average performance (around $80 \%$ ) on neutral reasoning tasks, our results showed that despite the low variability, individual differences in mindware instantiation were still strongly related to the overall conflict reasoning accuracy, and to a more moderate extent with one's conflict detection ability.

The link between conflict detection, mindware instantiation, and conflict reasoning accuracy may not be that surprising as the three factors are all indexed by very similar tasks, i.e., conflict, no-conflict, and neutral versions of the same reasoning problems. However, mindware instantiation was also moderately related to all of the standard individual difference predictors. This further supports the view that the differences between participants in 
available mindware are indeed meaningful, even if the variations in neutral reasoning problem accuracy are not large. Taken together, our results suggest that mindware instantiation may be a more important source of individual differences in conflict detection and overall reasoning accuracy than previously thought, and it should receive more attention in research on cognitive biases (see also Stanovich, 2018).

Along with mindware instantiation, several standard individual difference predictors contributed consistently to both detection efficiency and overall conflict reasoning accuracy. Their relationship with conflict detection ability in the present study was by and large consistent with the partial results presented by Pennycook et al. (2014, 2015), who have shown that both cognitive reflection and thinking dispositions are related to detection ability in the base-rate neglect task. Both of the aforementioned variables can be thought of as indicators of the propensity to recognize when one's intuitive thinking may be insufficient and more effortful processing is needed (Frederick, 2005; Stanovich et al., 2016), which might explain their contribution to one's conflict detection ability. Also, in line with Swan et al. (2018; however see Thompson \& Johnson, 2014), we have observed a weak correlation between cognitive ability and the confidence and latency detection efficiencies, but this relationship did not hold in the regression where other predictors were taken into account.

Whereas standard individual difference predictors played a relatively modest role in the conflict detection ability, they had a much more significant contribution to participant's overall conflict reasoning accuracy. Our results in this regard again concur with other studies which simultaneously examined several variables related to reasoning performance and found that cognitive ability, thinking dispositions, and numeracy or cognitive reflection are all independent predictors of conflict reasoning accuracy (Klaczynski, 2014; Toplak et al., 2011). The present research, however, brings a more comprehensive analysis which also takes into account estimates of participants' conflict detection ability and mindware instantiation, which showed up to predict reasoning over and above the standard individual difference predictors. Together, the full regression model accounted for $40 \%$ of the variance in conflict reasoning accuracy. This result, however, stands in sharp contrast with the predictive power of the regression models pertaining to conflict detection which only accounted for $7 \%$ of the variance in confidence detection efficiency, and even less in the efficiency index based on participant's response latencies. We discuss possible reasons for this difference later below. 
When analyzing individual differences in conflict detection, we have found some inconsistencies in the results pertaining to different detection efficiency indices. By and large, the index based on response latencies consistently yielded far less clear patterns of results than the one based on confidence. In comparison with the confidence index, the latency detection efficiency was not significantly related to mindware instantiation (i.e., the most potent predictor in the present research), its correlations with standard individual difference predictors were generally low, and the regression model with all variables explained only $2 \%$ of variance in this detection efficiency index. A possible explanation for this is the relative noisiness of the response latency measure. While reasoning problems were presented to participants in several parts to disentangle reading and decision latency as much as possible (e.g., Frey et al., 2018), the timing on the tasks was not restricted. This, together with the fact that wording of some reasoning problems is still quite lengthy (e.g., Pennycook et al., 2015), could result in larger variations in response time measurement rendering this method noisy. Also, as can be seen from the analyses presented in the supplementary materials, the results pertaining to the confidence latency measure were quite distinct from the other two detection indices. It was not related to any of the standard individual difference predictors, mindware instantiation, nor accuracy on conflict reasoning problems. Moreover, it showed almost no relation to the two other detection efficiencies, and also generally produced weaker detection effects than response latency and confidence measures. Taken together with the conclusions presented by Frey et al. (2018), these results strongly suggest that confidence latency is not a reliable indicator of the detection ability. Therefore, we would like to warn researchers to be careful when employing this measure in future conflict detection studies.

In line with previous studies, the present results also point to the limited domain generality of conflict detection (Frey \& De Neys, 2017; Frey et al., 2018). We observed quite some variability in the conflict detection efficiency across various reasoning problems. This means that even if someone is capable of registering conflicts on certain problems, they cannot be expected to also show more successful detection in other tasks. The low generality of conflict detection ability may well be the key reason for why we also obtained very low internal consistency estimates of conflict detection indices. Despite our effort to use the most robust way of measuring individual differences in conflict detection ability by employing four different reasoning problems and three detection indices, resulting detection efficiency estimates were far below satisfactory reliability. This certainly means that caution is needed 
when interpreting the results of conflict detection analyses and that our results will need to be replicated before drawing strong conclusions.

And yet, observed relationships between conflict detection indices, reasoning performance, and standard individual predictors, were quite in line with both previous partial research findings (Frey et al., 2018; Pennycook et al., 2014, 2015), and theoretical predictions regarding detection ability as important component of bias susceptibility (De Neys \& Bonnefon, 2013; Pennycook et al., 2015; Stanovich, 2018). Also, the fact that detection efficiencies did show up to predict conflict reasoning accuracy over standard individual difference predictors and mindware instantiation suggests that detection indices did capture some meaningful variance which is predictive of reasoning performance and is not due to other related cognitive factors which were included in the analysis. Most importantly, a recent study by Burič and Šrol (2019) offers some evidence for the replicability of the present conflict detection findings and thus lends further credence to the results we report here. Although their research was not designed as a direct replication of our study, the authors measured conflict detection as we did here. Their results show similar patterns of individual differences in conflict detection efficiency related to cognitive reflection and mindware instantiation and replicate the predictive role of detection efficiency and mindware instantiation in reasoning accuracy observed on conflict syllogisms and base-rate neglect tasks.

It should be noted, however, that Burič and Šrol (2019) also identified the problem with the low reliability of the conflict detection indices. We believe this low reliability is the key reason for why we have only managed to explain $7 \%$ of the variance in the confidence detection efficiency, even though we used a wide range of standard individual difference predictors as well as mindware instantiation as possible predictors. Given the low intercorrelations of detection efficiency between specific reasoning domains, any model examining general detection ability across tasks probably should not be expected to explain too much of the common variance. In the same way, low internal consistency has to be borne in mind when considering why some of the conceptually relevant variables did not show any relationship or were only modestly correlated with conflict detection, especially in the analyses pertaining to the latency detection index.

Taking into the account the low reliability of the conflict detection indices, relatively low correlations between detection effects observed across different tasks and measures, and 
overall low observed effects in the conflict detection analyses, it seems that further research on individual differences in the conflict detection mechanism will face a challenging task to overcome the issues identified in the present study. Still, it is clear that individual differences in conflict detection should not be disregarded, despite the problems the measurement of these differences may bear.

At this point, we would like to discuss some more theoretical implications of our findings for ongoing developments in the field of dual process theories of reasoning. As we mentioned, the conflict detection findings have led to a reformulation of traditional reasoning theories by positing that type 1 processing cues multiple intuitive responses based both on heuristics and the logical structure of the task (De Neys, 2012, 2013, 2017; Pennycook et al., 2015; Thompson \& Newman, 2017). Thus, what people are actually detecting in reasoning tasks is a conflict between two competing types of intuitions - one heuristic and the other logical. As such, the conflict detection mechanism is viewed under the recent hybrid dual process models to be a result of type 1 processing (De Neys, 2017; Pennycook, et al., 2015). Further, the likelihood of conflict detection and subsequent engagement in analytic type 2 processing has been thought to be determined by the relative strength of the logical and heuristic intuition. Specifically, it is assumed that conflict detection likelihood will be maximal when the strength of the two intuitively cued outputs is maximally similar (Bago \& De Neys, 2019a; De Neys \& Pennycook, 2019; Pennycook et al., 2015). However, as for most biased reasoners the heuristic intuition will be typically stronger than the logical one, correct responding on conflict reasoning tasks for them will require the analytic type 2 processing to override the dominant heuristic intuition.

One striking discrepancy in our results is that our predictors explained a much larger proportion of the variance in reasoning accuracy than in conflict detection. This may be due to the fact that reasoning accuracies observed on different problems are at least moderately intercorrelated (see table S2 in the supplementary material), which suggest more domain generality for overall reasoning accuracy than for conflict detection ability per se. In theoretical terms, this might imply that the type 2 process override success or capacity is more invariant across reasoning tasks than the type 1 detection component of the model. As reasoning performance is presumed to depend not only on efficient detection, but also intuition inhibition (De Neys \& Bonnefon, 2013; Pennycook et al., 2015; Stanovich, 2018), it might be that the latter process is more domain general than the former. 
More specifically, given that the conflict detection likelihood is assumed to depend on the relative strength of one's heuristic and/or logical intuitions (Pennycook et al., 2015; Bago \& De Neys, 2019a; De Neys \& Pennycook, 2019), these relative strengths might show much more variability across different reasoning tasks and/or even across different item contents of a single task. As is clear from the traditional group and individual-level conflict detection analyses both in the present study and in previous works (e.g., Frey \& De Neys, 2017; Frey et al., 2018), there is quite some variability in detection effects observed across different tasks. Moreover, even relatively minor changes to the specific features within the same reasoning task, such as manipulating the extremity of base-rates or the overall order of presented information within the reasoning problem, have been shown to influence the likelihood of conflict detection (Pennycook et al., 2012; 2015), presumably by differently increasing the strength of either the heuristic or logical intuition. Obviously, if the likelihood of conflict detection is determined by the difference in strength between the logical and heuristic intuitions and the strength of those intuitions varies even with small changes within the reasoning task, it is unlikely that conflict detection effectiveness would be very stable across problems with different formats and item contents. We speculate that it might be precisely this sensitivity of type 1 processing which leads to low domain generality of conflict detection indices and the associated methodological problems identified in the present research.

Although speculative, we believe that the difference between more context dependent intuitive type 1 processing in comparison with more domain general type 2 processing may help to account for the large observed gap between explained variance in detection efficiency and overall reasoning accuracy. Of course, this also suggests that currently it will be hard to study individual predictors of conflict detection, as researchers will have to deal with a substantial within and across task variability stemming from the type 1 processing. While we cannot currently offer any definitive solutions for the problems presented here, we hope that the analysis might still help to raise awareness of the issues that might complicate future research on individual differences in conflict detection.

Certainly, our study is not without its limitations. For one, our mindware instantiation index, which turned up to be the most consistent predictor in the present study, was of poor reliability. As with conflict detection, mindware instantiation has been argued to be considerably subject and task specific (Stanovich, 2018). This, coupled with the participant's ceiled performance on neutral reasoning problems may have led to the poor reliability of the mindware instantiation measure. Nevertheless, we have observed stable patterns of 
correlations between mindware instantiation and both conflict reasoning accuracy and standard individual difference predictors. As a side note, this may be also due to the fact that Cronbach's $\alpha$ represents an estimate of the lower bound of reliability of a given measure (Mair, 2018). Still, the low reliability of the mindware instantiation index points to the need to replicate the present results. It would be worthwhile to dedicate further effort to try to come up with a more reliable approach to measure participant's mindware instantiation, which would allow to better study this neglected component of cognitive bias research (Stanovich, 2018).

As one reviewer noted, since one of the main problems with our mindware instantiation measure lied in participants' very high average performance, one possible solution would be to increase the difficulty of neutral reasoning problems. However, we believe that such a solution comes with its own limitations. Specifically, while more complex neutral problems may better tap participants' potential, they will no longer represent the specific mindware needed to solve the less complex no-conflict and conflict versions. Still, it is possible that a simple solution such as increasing the number of neutral items might help researchers in future studies to limit the problem of low internal consistency and thus increase the reliability of findings pertaining to the role of the mindware component in the reasoning process. Although the mindware instantiation in our study showed up to be the most substantial predictor of both conflict detection and overall accuracy on conflict reasoning problems, due to low reliability of our mindware measure, the results will have to be taken with some caution (although, for similar patterns of results pertaining to mindware instantiation, see Burič \& Šrol, 2019).

Secondly, in our choice of standard individual predictor measures, we have relied on relatively short tests intended to tap the constructs of interest with only several items. This was done mainly because we wanted to reduce participants' fatigue resulting from the length of study (which was already exacerbated by the fact that we employed four types of problems to study reasoning accuracy and conflict detection). Future research might want to supplement our choice of predictor measures with longer, more reliable scales, as well as methods tapping into other constructs which have been previously shown to predict conflict reasoning accuracy, such as verbal intelligence, and/or actively open-minded thinking disposition (Stanovich et al., 2016). 
Individual difference studies are an integral part of the research on cognitive biases and have been paramount in advancing our understanding of the processes which are implicated in sound reasoning and decision-making (Stanovich \& West, 2008; Stanovich et al., 2016; Teovanović et al., 2015). While up till now the studies have uncovered several standard individual difference predictors which independently predict accuracy on conflict reasoning problems (e.g., Klaczynski, 2014; Toplak et al., 2011), they failed to relate these factors to specific components of bias susceptibility (De Neys \& Bonnefon, 2013; Pennycook et al., 2015; Stanovich \& West, 2008). In the present study, we set out to fill this gap by examining individual difference predictors specifically related to one of these components, the ability to detect a conflict between intuition and logic, and determine how these differences relate to overall accuracy on reasoning problems. We have found that while several standard individual difference predictors contributed to various extent to both conflict detection and reasoning accuracy, the most important factor in both regards showed up to be mindware instantiation. Mindware instantiation has long been recognized as a theoretically critical component of sound reasoning (Stanovich, 2018; Stanovich \& West, 2008). However, up until now, it was mostly neglected in the empirical research in this area. Thus, the present study highlights the importance of teasing apart specific components of sound reasoning and studying their relative contributions to overall susceptibility to cognitive biases.

\section{References}

Bago, B., \& De Neys, W. (2019a). Advancing the specification of dual process models of higher cognition: A critical test of the hybrid model view. Thinking \& Reasoning, http://dx.doi.org/10.1080/13546783.2018.1552194.

Bago, B., Frey, D., Vidal, J., Houdé, O., Borst, G., \& De Neys, W. (2018). Fast and slow thinking: Electrophysiological evidence for early conflict sensitivity. Neuropsychologia, 117(June), 483-490.

Burič, R., \& Šrol, J. (2019). Individual differences in logical intuitions on reasoning problems presented under two-response paradigm. https://doi.org/10.31234/osf.io/w6un2

Cokely, E. T., Galesic, M., Schulz, E., Ghazal, S., \& Garcia-Retamero, R. (2012). Measuring Risk Literacy: The Berlin Numeracy Test. Judgment and Decision Making, 7(1), 25-47. 
De Neys, W. (2012). Bias and Conflict: A Case for Logical Intuitions. Perspectives on Psychological Science, 7(1), 28-38.

De Neys, W. (2013). Conflict detection, dual processes, and logical intuitions: Some clarifications. Thinking \& Reasoning, 20, 1-19.

De Neys, W. (2017). Bias, conflict, and fast logic: towards a hybrid dual process future? In W. De Neys (Ed.), Dual process theory 2.0 (pp. 47-65). London: Routledge.

De Neys, W., \& Bonnefon, J.-F. (2013). The "whys" and "whens" of individual differences in thinking biases. Trends in Cognitive Sciences, 17(4), 172-178.

De Neys, W., \& Glumicic, T. (2008). Conflict monitoring in dual process theories of thinking. Cognition, 106(3), 1248-1299.

De Neys, W., Moyens, E., \& Vansteenwegen, D. (2010). Feeling we're biased: autonomic arousal and reasoning conflict. Cognitive, Affective \& Behavioral Neuroscience, 10(2), 208-216.

De Neys, W., \& Pennycook, G. (2019). Logic, fast and slow: Advances in dual-process theorizing. Current Directions in Psychological Science, 0963721419855658.

De Neys, W., Rossi, S., \& Houdé, O. (2013). Bats, balls, and substitution sensitivity: cognitive misers are no happy fools. Psychonomic Bulletin \& Review, 20(2), 269-73.

De Neys, W., Vartanian, O., \& Goel, V. (2008). Smarter Than We Think. Psychological Science, 19(5), 483-489.

Epstein, S., Pacini, R., Denes-Raj, V., \& Heier, H. (1996). Individual differences in intuitiveexperiential and analytical-rational thinking styles. Journal of Personality and Social Psychology, 71(2), 390-405.

Evans, J. S. B. T. (2007). On the resolution of conflict in dual process theories of reasoning. Thinking \& Reasoning, 13(4), 321-339.

Evans, J. S. B. T., \& Stanovich, K. E. (2013). Dual-Process Theories of Higher Cognition: Advancing the Debate. Perspectives on Psychological Science, 8(3), 223-241.

Fagerlin, A., Zikmund-Fisher, B. J., Ubel, P. A., Jankovic, A., Derry, H. A., \& Smith, D. M. (2007). Measuring Numeracy without a Math Test: Development of the Subjective 
Numeracy Scale. Medical Decision Making, 27(5), 672-680.

Franssens, S., \& De Neys, W. (2009). The effortless nature of conflict detection during thinking. Thinking \& Reasoning, 15(2), 105-128.

Frederick, S. (2005). Cognitive Reflection and Decision Making. Journal of Economic Perspectives, 19(4), 25-42.

Frey, D., \& De Neys, W. (2017). Is Conflict Detection in Reasoning Domain General ? In Proceedings of the Annual Meeting of the Cognitive Science Society, 39 (pp. 391-396).

Frey, D., Johnson, E. D., \& De Neys, W. (2018). Individual differences in conflict detection during reasoning. The Quarterly Journal of Experimental Psychology, 71(5), 1188-1208.

Kahneman, D. (2011). Thinking, fast and slow. New York: Penguin Books.

Klaczynski, P. A. (2014). Heuristics and biases: Interactions among numeracy, ability, and reflectiveness predict normative responding. Frontiers in Psychology, 5(JUL), 1-13.

Klose, J., Černochová, D., \& Král, P. (2002). Videňský maticový test. Praha: Testcentrum.

Mair, P. (2018). Modern Psychometrics with R. New York: Springer.

Mata, A., Ferreira, M. B., Voss, A., \& Kollei, T. (2017). Seeing the conflict : an attentional account of reasoning errors. Psychonomic Bulletin \& Review, 24(6), 1980-1986.

Mevel, K., Poirel, N., Rossi, S., Cassotti, M., Simon, G., Houdé, O., \& De Neys, W. (2014). Bias detection: Response confidence evidence for conflict sensitivity in the ratio bias task. Journal of Cognitive Psychology, 27(2), 227-237.

Pennycook, G., Cheyne, J. A., Barr, N., Koehler, D. J., \& Fugelsang, J. A. (2014). Cognitive style and religiosity: the role of conflict detection. Memory \& Cognition, 42(1), 1-10.

Pennycook, G., Fugelsang, J. A., \& Koehler, D. J. (2012). Are we good at detecting conflict during reasoning? Cognition, 124(1), 101-6.

Pennycook, G., Fugelsang, J. A., \& Koehler, D. J. (2015). What makes us think? A threestage dual-process model of analytic engagement. Cognitive Psychology, 80, 34-72.

Singmann, H., Klauer, K. C., \& Kellen, D. (2014). Intuitive logic revisited: New data and a bayesian mixed model meta-analysis. PLoS ONE, 9(4), e94223. 
Stanovich, K. E. (2018). Miserliness in human cognition: The interaction of detection, override and mindware. Thinking \& Reasoning. http://doi.org/10.1080/13546783.2018.1459314

Stanovich, K. E., \& West, R. F. (2008). On the relative independence of thinking biases and cognitive ability. Journal of Personality and Social Psychology, 94(4), 672-695.

Stanovich, K. E., West, R. F., \& Toplak, M. E. (2016). The Rationality Quotient: Toward a test of rational thinking. MIT Press.

Stupple, E. J., Ball, L. J., \& Ellis, D. (2013). Matching bias in syllogistic reasoning: Evidence for a dual- process account from response times and confidence ratings. Thinking \& Reasoning, 19(1), 54-77.

Stupple, E. J., \& Ball, L. J. (2008). Belief-Logic Conflict Resolution in Syllogistic Reasoning: Inspection-Time Evidence for a Parallel-Process Model. Thinking Reasoning, 14(2), $168-181$.

Swan, A. B., Calvillo, D. P., \& Revlin, R. (2018). To detect or not to detect: A replication and extension of the three-stage model. Acta Psychologica, 187(October 2017), 54-65.

Šrol, J. (2019). Individual differences in susceptibility to cognitive biases: implication for theories of rational thought. Unpublished doctoral thesis, Slovak Academy of Sciences, Bratislava, SK.

Teovanović, P., Knežević, G., \& Stankov, L. (2015). Individual differences in cognitive biases: Evidence against one-factor theory of rationality. Intelligence, 50, 75-86.

Thompson, V. A., \& Johnson, S. C. (2014). Conflict, metacognition, and analytic thinking. Thinking \& Reasoning, 20(January 2015), 215-244.

Thompson, V. A., \& Newman, I. R. (2017). Logical intuitions and other conundra for dual process theories. In W. De Neys (Ed.), Dual process theory 2.0 (pp. 121-136). London: Routledge.

Thomson, K. S., \& Oppenheimer, D. M. (2016). Investigating an alternate form of the cognitive reflection test. Judgment and Decision Making, 11(1), 99-113.

Toplak, M. E., West, R. F., \& Stanovich, K. E. (2011). The Cognitive Reflection Test as a 
predictor of performance on heuristics-and-biases tasks. Memory \& Cognition, 39(7), $1275-1289$.

Tversky, A., \& Kahneman, D. (1983). Extensional Versus Intuitive reasoning: The conjunction fallacy in probability judgment. Psychological Review, 90(4), 293-315.

Vartanian, O., Beatty, E. L., Smith, I., Blackler, K., Lam, Q., Forbes, S., \& De Neys, W. (2018). The Reflective Mind: Examining Individual Differences in Susceptibility to Base Rate Neglect with fMRI. Journal of Cognitive Neuroscience, 30(7), 1-12. 